\title{
Loculated Empyema and SARS-CoV-2 Infection: A Report of Two Cases and Review of the Literature
}

\author{
$\underline{\text { Sarah Ayad }}^{1}$, Kirolos Gergis², Sherif Elkattawy ${ }^{1}$, Noreen Mirza ${ }^{3}$, Basel Abdelazeem ${ }^{2}$, Latika Patel ${ }^{1}$, Carlos Remolina $^{4}$ \\ ${ }^{1}$ Internal Medicine, Rutgers-New Jersey Medical School/Trinitas Regional Medical Center, Elizabeth, New Jersey, USA \\ ${ }^{2}$ Internal Medicine, McLaren Health Care, Flint, Michigan, USA \\ ${ }^{3}$ St. George's University, Grenada, West Indies \\ ${ }^{4}$ Department of Pulmonology, Trinitas Regional Medical Center, Elizabeth, New Jersey, USA
}

\section{Doi: 10.12890/2021_002706 - European Journal of Case Reports in Internal Medicine - @ EFIM 2021}

Received: 10/06/2021

Accepted: $14 / 06 / 2021$

Published: $15 / 07 / 2021$

How to cite this article: Ayad S, Gergis K, Elkattawy S,Mirza N, Abdelazeem B, Patel L, Remolina C. Loculated empyema and SARS-CoV-2 infection: a report of two cases and review of the literature. EJCRIM 2021;8: doi:10.12890/2021_002707.

Conflicts of Interests: The authors declare there are no competing interests.

This article is licensed under a Commons Attribution Non-Commercial 4.0 License

\section{ABSTRACT}

Coronavirus disease 2019 (COVID-19) is caused by severe acute respiratory syndrome coronavirus 2 (SARS-CoV-2). Clinical manifestations are diverse and can vary from mild respiratory symptoms to severe hypoxic respiratory failure. In severe cases, infection can cause gastrointestinal, renal, cardiac, neurological and haematological complications and result in multi-organ failure. There are very few reports of parapneumonic effusion in patients with COVID-19. We describe two patients with COVID-19 who had loculated empyema and discuss the clinical course and therapeutic options.

\section{LEARNING POINTS}

- The clinical manifestations of COVID-19 vary from mild to severe disease and can result in multi-organ failure.

- Pleural empyema is usually treated with a combination of antibiotics and surgical drainage of the pleural cavity.

\section{KEYWORDS}

Empyema, loculated pleural effusion, COVID-19, SARS-CoV-2

\section{INTRODUCTION}

Coronavirus disease 2019 (COVID-19), caused by severe acute respiratory syndrome coronavirus 2 (SARS-CoV-2), was first described in Wuhan, China in December 2019 and has since developed into a global pandemic with serious psychological and economic consequences ${ }^{[1]}$. As of June 2021, over 173 million cases have been confirmed, over 3.7 million people have died, and over 1.9 billion doses of vaccine have been administered ${ }^{[2]}$.

COVID-19 symptoms typically affect the respiratory system and include cough, fever, shortness of breath and fatigue ${ }^{[3]}$. The symptoms may be serious enough to cause hospitalization and ventilator-dependent hypoxic respiratory failure, and can lead to multi-organ failure in severe cases.

Parapneumonic effusion, a life-threatening condition, is a rare complication of pneumonia, accounting for about 2-3\% of all pneumonias, with numbers steadily increasing over time. To date, there is very little in the literature on parapneumonic effusions in patients with COVID-19 ${ }^{[4,5]}$. We describe two patients with COVID-19 who were found to have loculated empyema. We will discuss the clinical course and therapeutic options. 


\section{CASE DESCRIPTION 1}

Our patient was a 60-year-old man with a medical history significant for poorly controlled type 2 diabetes mellitus and hypertension, who presented to the emergency room after sustaining a mechanical fall. The patient disclosed a 5-day history of general malaise, asthenia, anorexia and subjective fevers. Symptoms were associated with progressively worsening right-sided, constant and pleuritic chest pain. Pain was $8 / 10$ in intensity, sharp in nature, and exacerbated with deep inspiration and coughing. On presentation, blood pressure was $164 / 84$ $\mathrm{mmHg}$, heart rate was 129 beats per minute, there were 40 breaths per minute, with oxygen saturation of $89 \%$ on room air, and temperature was $38.3^{\circ} \mathrm{C}$. Physical examination revealed a male on oxygen supplementation via a nasal cannula who was in apparent respiratory distress. Lung examination was significant for absent breaths sounds in the right base of the lung; left lung breaths sounds were present.

A nasopharyngeal swab was positive for SARS-CoV-2. Laboratory examinations were significant for white blood count (WBC) $11.8 \mathrm{~K} / \mu \mathrm{l}$ (4.8-10.8), haemoglobin 14.1 g/dl (14-18), platelets $823 \mathrm{~K}$ (130-400), lymphocytes 6.0\% (20.5-51.1\%), absolute lymphocytes $0.7 \mathrm{~K} / \mu \mathrm{l}$ (1.23.4), LDL $182 \mathrm{U} / \mathrm{I}$ (98-192), ferritin $540 \mathrm{ng} / \mathrm{ml}$ (23.9-336.2), D-dimer $882 \mathrm{ng} / \mathrm{ml}$ (0-230) and fibrinogen >800 mg/dl (270-500).

A chest $x$-ray (CXR) showed a large right pneumothorax with near complete collapse of the right lung, and mediastinal shift to left (Fig. $1 A$ ). A cardiothoracic surgeon was consulted, an emergent pigtail thoracostomy tube was placed and a large amount of milky brown fluid was drained. Repeat CXR showed partial re-aeration of the right lung following placement of the chest tube and return of the mediastinum to the neutral position (Fig. 1B).
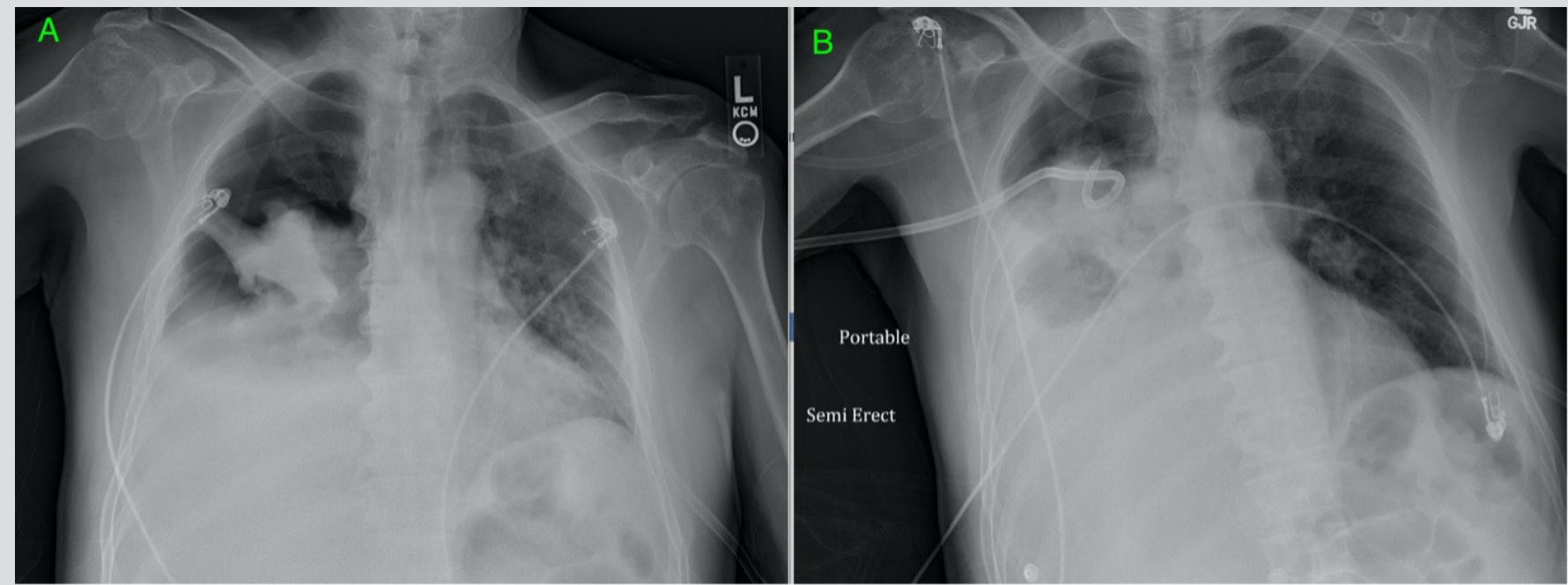

Figure 1. (A) Chest $x$-ray showing a large right pneumothorax with near complete collapse of the right lung, and mediastinal shift to the left. (B) Repeat chest $x$-ray showing partial re-aeration of the right lung following placement of a chest tube and return of the mediastinum to the neutral position

A CT pulmonary angiogram did not show any pulmonary emboli, but did show a large right hydropneumothorax, complete collapse of the right middle and lower lobes, and partial collapse of the right upper lobe (Fig. 2). Because of the incomplete resolution of the pneumothorax, interventional radiology was asked to increase the diameter of the catheter, and a second pigtail catheter was placed. The patient was admitted to the intensive care unit for close monitoring. He was started on intravenous vancomycin $1 \mathrm{~g}$ every $12 \mathrm{~h}$ and intravenous piperacillin/tazobactam 3.375 g every 8 hours pending sensitivity results. Chest tube fluid analysis and cultures were significant for: fluid appearance: brown, turbid; fluid WBC: 251,600 [/CMM]; fluid RBC: 64,160; fluid protein: $1.2 \mathrm{~g} / \mathrm{dl}$; fluid LDH: 695 /l; fluid glucose: 16 mg/ dl; fluid culture: many Streptococcus anginosus; serum LDH: $182 \mu / \mathrm{l}$ serum protein: $0.5 \mathrm{~g} / \mathrm{dl}$.

Analysis of the patient's pleural fluid was consistent with exudative effusion. Antibiotics were switched to intravenous ceftriaxone 2000 $\mathrm{mg}$ daily and oral metronidazole $500 \mathrm{mg}$ every 8 hours for empyema with S. anginosus. The patient was also started on the MIST protocol for intrapleural instillation of tissue plasminogen activator (tPA) and dornase alfa for loculated empyema, which was repeated over 3 days. On day 10 of hospitalization, the anterior pigtail was removed, and the lateral pigtail was left in place. The patient underwent repeat CT which showed residual loculated empyema at the very inferior posterior aspect of the right pleural space measuring $9 \times 9 \times 3 \mathrm{~cm}$, somewhat more superiorly and medially, a second collection measuring $9 \times 9 \times 6 \mathrm{~cm}$, and a small residual collection in the right major fissure measuring $2.5 \mathrm{~cm}$ (Fig. 3). The patient underwent CT-guided evacuation of the loculated pneumothorax and insertion of a chest tube. He continued to 
improve clinically, and the chest tubes were subsequently removed. A repeat CXR is seen in Fig. 4. The patient was deemed a good candidate for outpatient follow-up and was discharged home on amoxicillin/clavulanic acid. $875 \mathrm{mg}$ twice a day for 7 days.

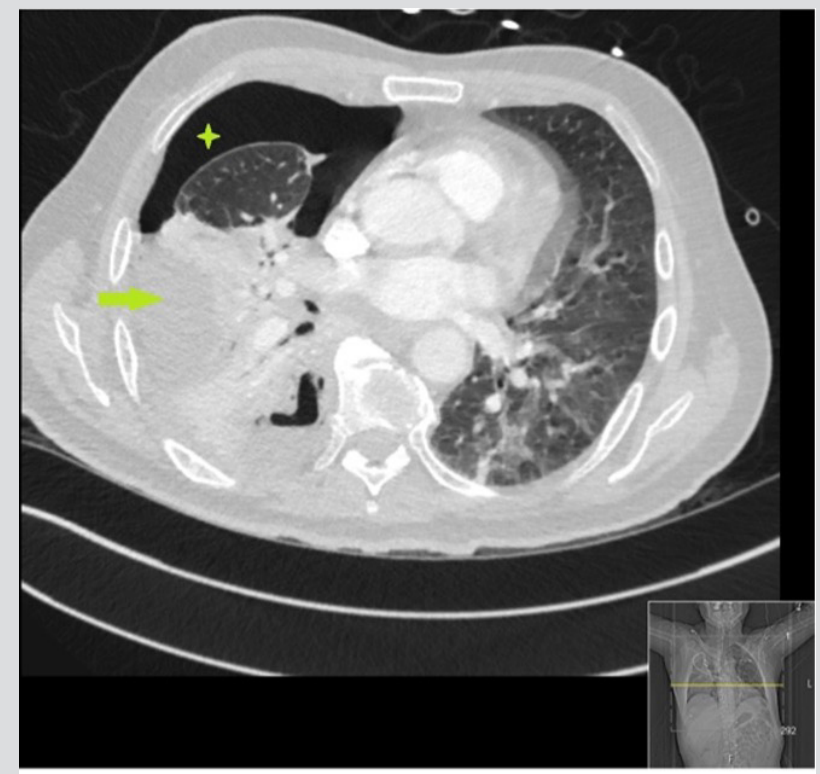

Figure 2. CT pulmonary angiogram showing a large right hydropneumothorax (arrow: fluid; star: air), with an apical chest tube in place, with complete collapse of the right middle and lower lobes and partial collapse of the right upper lobe

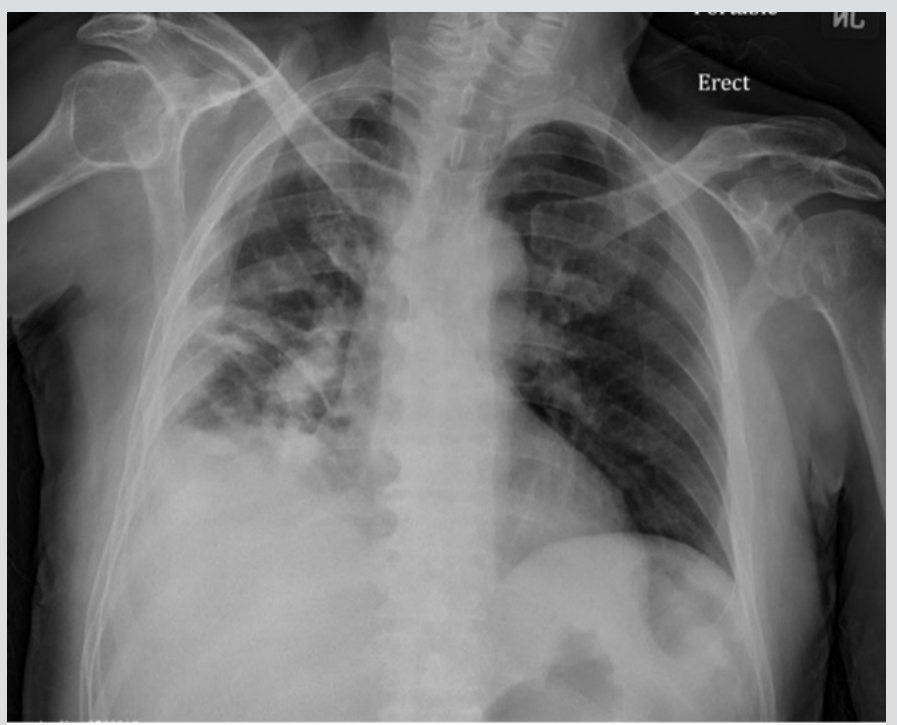

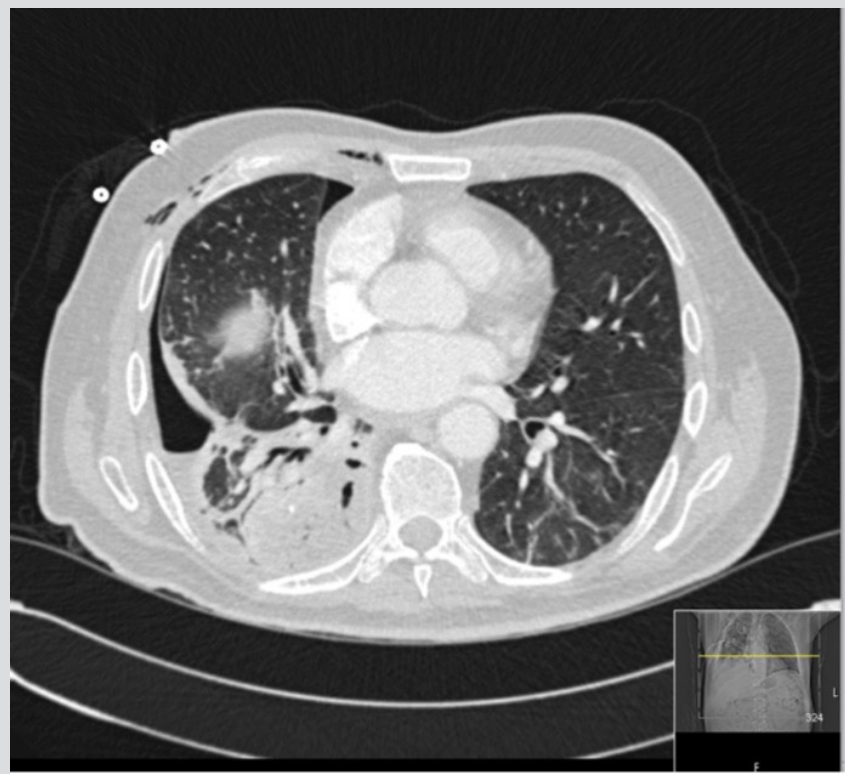

Figure 3. Repeat CT showing residual loculated empyema at the very inferior posterior aspect of the right pleural space

Figure 4. Repeat chest $\mathrm{x}$-ray prior to discharge

\section{CASE DESCRIPTION 2}

A 73-year-old Hispanic man with a medical history of recent COVID-19 pneumonia, pulmonary abscess, pulmonary embolism, diabetes mellitus and hypertension presented to the emergency department from a nursing home for evaluation of low haemoglobin levels. On presentation, he was complaining of non-productive cough and shortness of breath, and was hypoxaemic on supplemental oxygen. He denied any blood per rectum, black tarry stools, haemoptysis or haematemesis. On presentation, his temperature was $37.3^{\circ} \mathrm{C}$, respiratory rate was 18 , blood pressure was $146 / 75 \mathrm{mmHg}$, heart rate was 117 , and oxygen saturation was $88 \%$ on a 3 -litre nasal cannula. Physical 
examination was unremarkable. Laboratory results were remarkable for haemoglobin $7.2 \mathrm{~g} / \mathrm{dl}$, platelets $269 \mathrm{~K}$ (130-400), WBC count 8.4 $\mathrm{K} / \mathrm{Ml}$ (4.8-10.8), lactic acid $1.21 \mathrm{mmol} / \mathrm{l}$ (0.5-2), INR 1.1, creatinine $0.7 \mathrm{mg} / \mathrm{dl}$ (0.7-1.2), sodium $134 \mathrm{mmol} / \mathrm{l}$ (136-144) and potassium 4.4 $\mathrm{mmol} / \mathrm{l}$ (3.6-5.1). A faecal occult blood test was negative. CXR showed worsening pneumonia (Fig. 5).

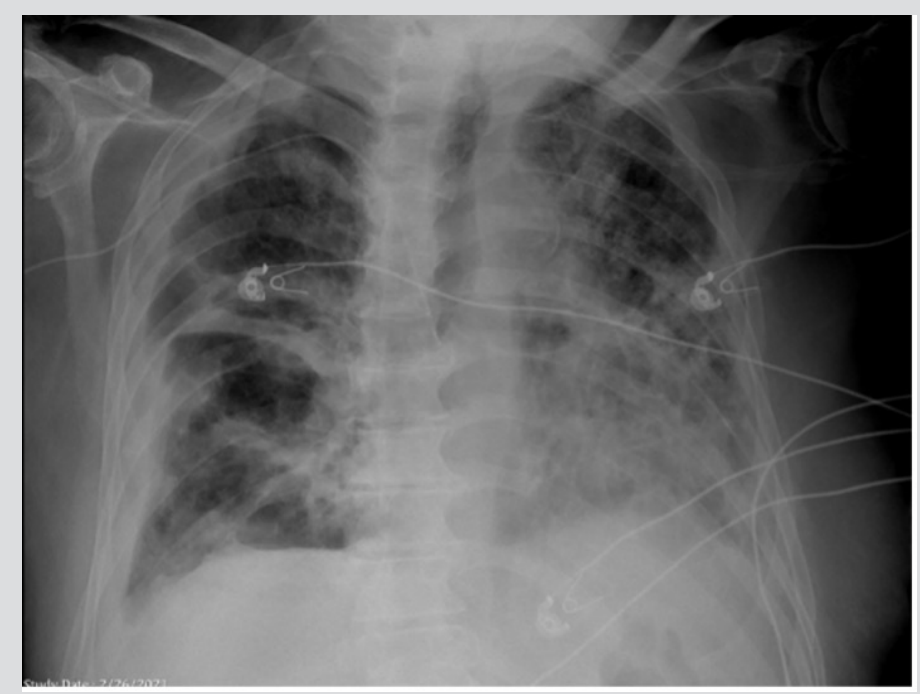

Figure 5. Chest $x$-ray showing worsening pneumonia

Of note, patient had been admitted 3 months previously for hypoxic respiratory failure secondary to COVID-19 pneumonia (and had required a high flow nasal cannula but was not intubated) and multiple pulmonary emboli. He was discharged on rivaroxaban to subacute rehabilitation and completed 10 days of remdesivir and dexamethasone. He was readmitted 2 weeks later with leucocytosis above 30,000 $\mathrm{K} / \mu \mathrm{l}$, progressive infiltrates on a CT scan of the chest, a small-to-moderate right-sided pleural effusion, and possibly a developing abscess at the lung base. Cultures were negative, he improved clinically, and leucocytosis resolved while he was on broad spectrum antibiotics. $A$ follow-up CT scan did not show further enlargement of the effusion but did show evolution into an area of abscess formation at the right lung base. A right-sided peripherally inserted central catheter (PICC) line was placed, and he was continued on antibiotic treatment on the advice of the infectious disease consultant given improvement seen on a repeat CT scan, and transferred back to rehabilitation.

During this admission, rivaroxaban was held, and the patient was given 1 unit of packed red blood cells with an appropriate haemoglobin response. He was started on broad-spectrum antibiotics for coverage including vancomycin and meropenem. A CT scan of the chest was significant for interval worsening, right lower lobe cavitary consolidation with apparent extension of the interval into the pleural fluid, and suspected associated right-sided empyema (Fig. 6).

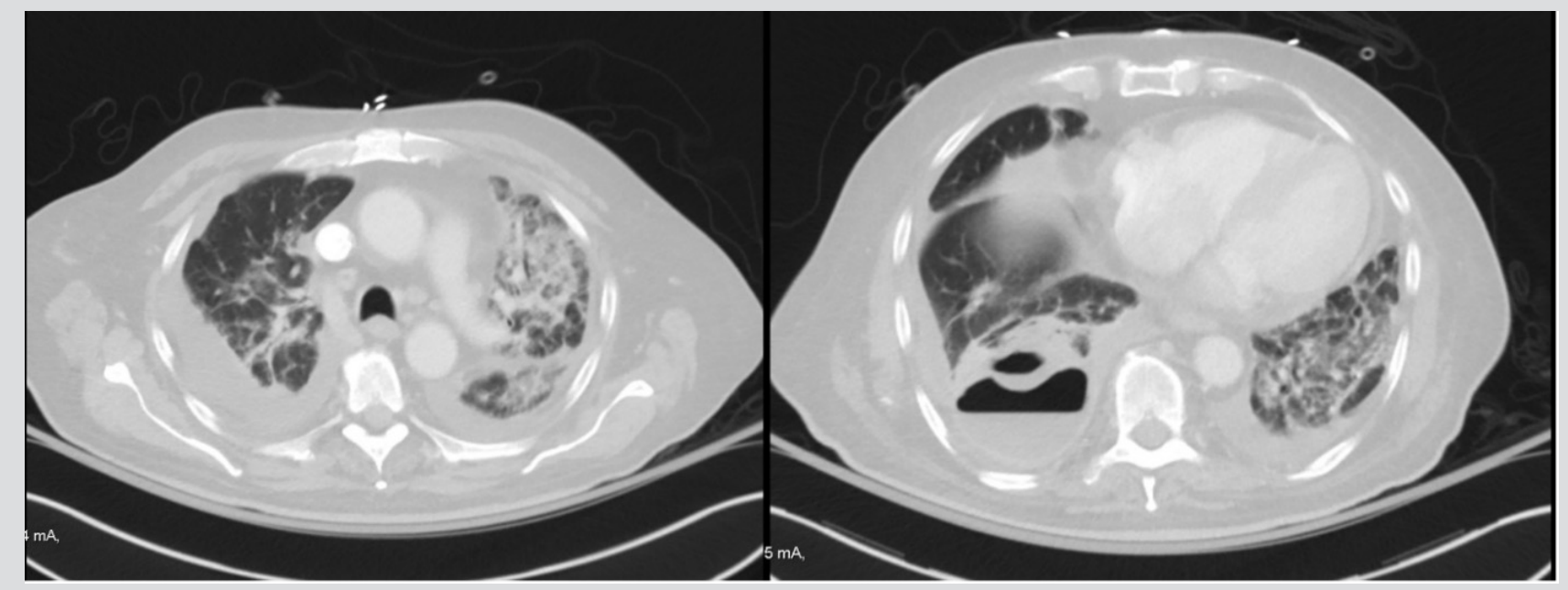

Figure 6 A CT scan of the chest was significant for interval worsening, right lower lobe cavitary consolidation with apparent extension of the interval into the pleural fluid and suspected associated right-sided empyema 
Interventional radiology was consulted for drainage of empyema. During insertion of the chest tube, the rapid response team was called when the patient became unresponsive. His blood sugar was found to be $25 \mathrm{mg} / \mathrm{dl}$. He was given dextrose $50 \times 2$ and glucagon $1 \mathrm{mg}$ with subsequent glucose increase to $>300 \mathrm{mg} / \mathrm{dl}$ and immediate improvement of mentation. He was transferred to the intensive care unit for observation. Blood and urine culture showed no growth. A repeat CT scan of the chest was performed after right chest tube placement and was significant for improvement of right-sided effusion and almost complete resolution of the right lower lobe abscess (Fig. 7). The chest tube was eventually removed. A transthoracic echocardiogram showed an ejection fraction of 55-60\% with trivial pericardial effusion present. Thoracocentesis was performed with the following pleural fluid results: lactate dehydrogenase $577 \mu / \mathrm{l}$, glucose $124 \mathrm{mg} / \mathrm{dl}$, protein $2.7 \mathrm{~g} / \mathrm{dl}, \mathrm{pH}$ 8.5, WBC 367 [/CMM], RBC 46,050 [/CMM], triglycerides 39 mg/dL, fluid differential: neutrophils 40\% and lymphocytes 20\%, and negative fungus, acid fast bacilli and fluid culture. Pleural fluid was determined to be transudative based on Light's criteria. While a specific pathogen was never identified, the patient had been on a prolonged antibiotic course before fluid analysis. The patient's oxygenation improved after thoracentesis and chest tube placement. His hospital stay was complicated with Clostridium difficile infection, which was treated with antibiotics. He also developed acute kidney injury, possibly due to vancomycin which was discontinued with improvement of renal function. The hospital stay was further complicated with urinary retention: he failed a trial of void and was discharged with a Foley catheter in place.

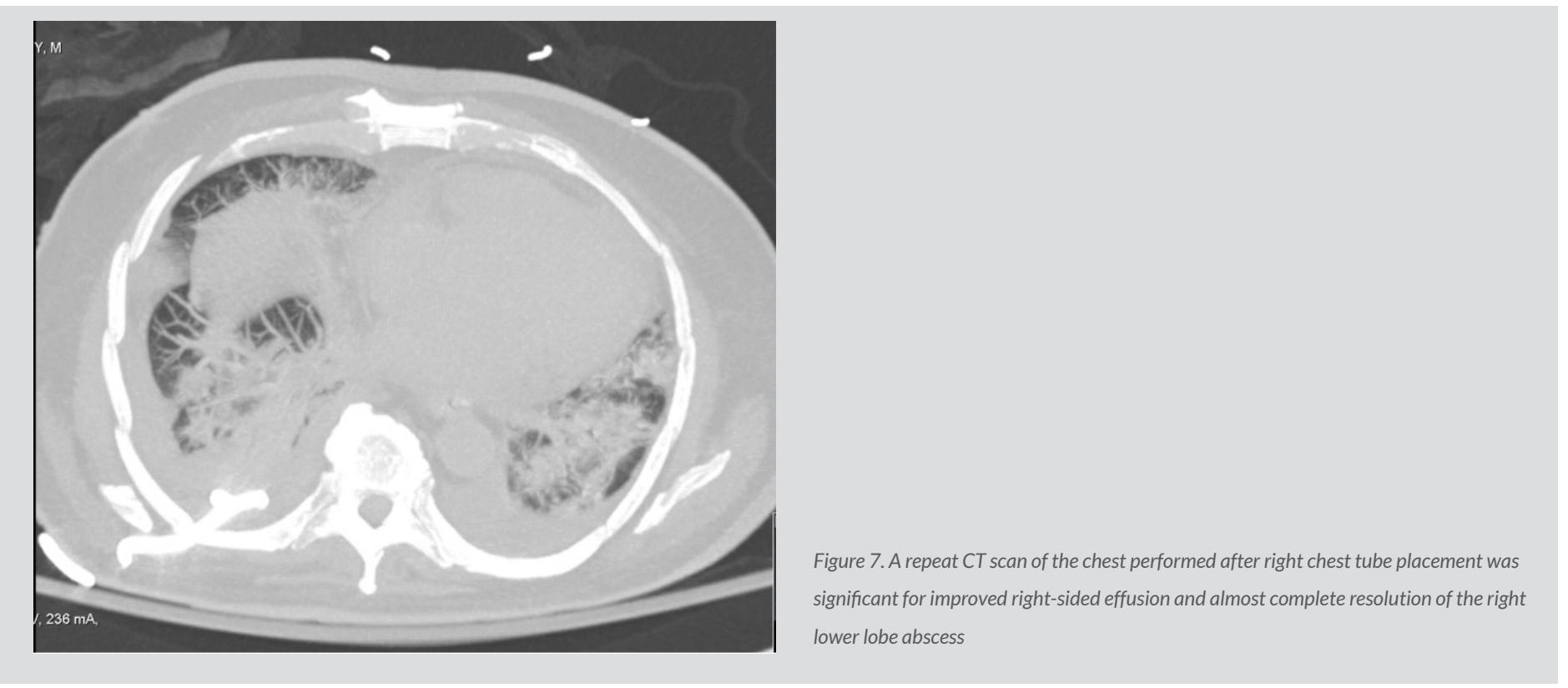

\section{DISCUSSION}

Many uncommon COVID-19 complications have been reported, including parapneumonic effusions, which can be categorized as uncomplicated, complicated or empyema thoracis. They are relatively uncommon complications of pneumonia, with rates of $2-3 \%$ of all pneumonias although the rates have been slowly rising over the years ${ }^{[4,5]}$. Hospitalized patients have the highest frequency of parapneumonic effusions.

Uncomplicated effusions consist of free-flowing sterile interstitial fluid caused by the inflammation associated with pneumonia. On thoracentesis drainage, the fluid is normally cloudy or clear in colour and generally resolves with the administration of antibiotics ${ }^{[6]}$.

Complicated parapneumonic effusions are due to invasion of the pleural space by bacteria or other microorganisms. Due to prompt clearance of bacteria, the fluid is also typically sterile and cloudy in colour. These effusions normally require drainage and antibiotics ${ }^{[6]}$.

Empyema thoracis results from the accumulation of pus when microorganisms like fungi, mycobacteria or pyogenic bacteria invade the pleural space, and is associated with high morbidity and mortality worldwide ${ }^{[7]}$. The fluid/pus is typically opaque, viscous and thick ${ }^{[6]}$. Empyema that develops from adjacent pneumonia is a subclass of complicated parapneumonic effusion. Potential sources of empyema include the common translocation of microorganisms from adjacent pneumonia, direct inoculation (surgical or non-surgical trauma), haematogenous spread, diaphragmatic spread of microorganisms from the abdomen, contiguous spread from a mediastinal infection, and reactivation of mycobacterial infections ${ }^{[6]}$.

Essentially, parapneumonic effusions can be caused by any microorganism that also causes pneumonia. Viral and mycoplasma pneumonia 
have been implicated in around $20 \%$ of cases of parapneumonic effusion. Streptococcus pneumoniae, Staphylococcus aureus and oral anaerobes are some of the common causes of parapneumonic effusion ${ }^{[6]}$. Tsang et al. found that the most common pathogen isolates from 63 patients with parapneumonic effusions were Streptococcus species (26\%), Bacteroides (14\%) and Klebsiella pneumoniae (12\%) ${ }^{[8]}$. The most common pathogens associated with empyema include the Streptococcus milleri group, Strep. pneumoniae, methicillin-sensitive Staph. aureus (MSSA) and the Enterobacteriaceae group ${ }^{[7]}$.

The chest CT in our patient was particularly important as it revealed empyema, a rare complications that should be considered in a COVID-19 patient who is not clinically improving. Some studies have investigated the frequency of parapneumonic effusions in COVID-19. In a metaanalysis, Chong et al. reviewed 47 observational studies with 4981 COVID-19 patients at risk of developing pleural effusion. They found a low incidence of $7.3 \%$ for parapneumonic pleural effusions which increased in those who were critically ill or had multisystem inflammatory syndrome ${ }^{[9]}$. They also suggested that the characteristic findings of COVID-19-related pleural effusion are exudative, lymphocytic or neutrophilic-predominant pleural fluid with markedly elevated LDH levels and a pleural fluid-to-serum (PF:S) LDH ratio of around 1.3. Furthermore, Zhang et al. examined 34 COVID-19 patients aged 7-88 years and found only one patient with pleural effusion ${ }^{[10]}$. Another study showed that the incidence of empyema increases over the course of hospital stay ${ }^{[11]}$. Thus, it is essential to consider empyema in patients who have long and complicated hospital stays.

Most parapneumonic effusions need to be sampled via thoracentesis unless the effusion is too small. Management is dependent on the results of fluid analysis from a diagnostic thoracentesis, which in a few cases, may also be therapeutic. If the parapneumonic effusion is small to moderate in volume, has a $\mathrm{pH}>7.30$, is free-flowing and is not purulent, it likely can be treated with antibiotics ${ }^{[6]}$. If the $\mathrm{pH}$ is $<7.20$ and there is loculated pleural fluid, pleural space drainage will probably be required. Guidelines for the management of empyema in COVID-19 have not yet been established. Tessitore et al. discussed three cases of pleural empyema after bilateral interstitial COVID-19 pneumonia which required combined medical and surgical treatment with open decortication ${ }^{[12]}$. The main treatment of pleural empyema is a course of a single or a combination of antibiotics. However, antibiotics alone are usually unsuccessful for pleural empyema and the majority of cases require surgical treatment with drainage of the pleural cavity ${ }^{[7]}$.

\section{CONCLUSIONS}

This report highlights the importance of rare complications such as empyema which may present after COVID-19 pneumonia and the need for continued monitoring for this complication in patients who fail to clinically improve during their hospital stay.

\section{REFERENCES}

1. Zhu H, Wei L, Niu P. The novel coronavirus outbreak in Wuhan, China. Glob Health Res Policy 2020;5(2):6. Available from: https://doi.org/10.1186/s41256-020-00135-6

2. World Health Organization. WHO Coronavirus (COVID-19) Dashboard. 2021. Available from: https://covid19.who.int/ (accessed 7 Jun 2021).

3. Placik DA, Taylor WL, Wnuk NM. Bronchopleural fistula development in the setting of novel therapies for acute respiratory distress syndrome in SARS-CoV-2 pneumonia. Radiol Case Rep 2020;15(11):2378-2381. Available from: https://doi.org/10.1016/j.radcr.2020.09.026

4. Weese WC, Shindler ER, Smith IM, Rabinovich S. Empyema of the thorax then and now. A study of 122 cases over four decades. Arch Intern Med 1973;131(4):516-520. Available from: http://archinte.jamanetwork.com/article.aspx?doi=10.1001/archinte.1973.00320100044004

5. Farjah F, Symons RG, Krishnadasan B, Wood DE, Flum DR. Management of pleural space infections: a population-based analysis. J Thorac Cardiovasc Surg 2007;133(2):346351. Available from: http://www.ncbi.nlm.nih.gov/pubmed/17258562

6. Sahn SA. Diagnosis and management of parapneumonic effusions and empyema. Clin Infect Dis 2007;45(11):1480-1486. Available from: http://www.ncbi.nlm.nih.gov/ pubmed/17990232

7. Marks DJB, Fisk MD, Koo CY, Pavlou M, Peck L, Lee SF, et al. Thoracic empyema: a 12-year study from a UK tertiary cardiothoracic referral centre. PLoS One 2012;7(1):e30074.

8. Tsang KY, Leung WS, Chan VL, Lin AWL, Chu CM. Complicated parapneumonic effusion and empyema thoracis: microbiology and predictors of adverse outcomes. Hong Kong Med J 2007;13(3):178-186.

9. Chong WH, Saha BK, Conuel E, Chopra A. The incidence of pleural effusion in COVID-19 pneumonia: state-of-the-art review. Heart Lung 2021;50(4):481-490. Available from: https://doi.org/10.1016/j.hrtIng.2021.02.015

10. Zhang L, Kong X, Li X, Zhu J, Liu S, Li W, et al. CT imaging features of 34 patients infected with COVID-19. Clin Imaging 2020;68(29):226-231. Available from: https://doi. $\operatorname{org} / 10.1016 /$ j.clinimag.2020.05.016

11. Guan CS, Wei LG, Xie RM, Lv ZB, Yan S, Zhang ZX, et al. CT findings of COVID-19 in follow-up: comparison between progression and recovery. Diagn Interv Radio 2020;26(4):301-307.

12. Tessitore A, Patella M, Giuliani M, Theologou T, Freguia S, Minerva EM, et al. Surgical treatment of pleural empyema in Coronavirus disease 19 patients: the Southern Switzerland experience. Interact Cardiovasc Thorac Surg 2021;32(3):367-370. 